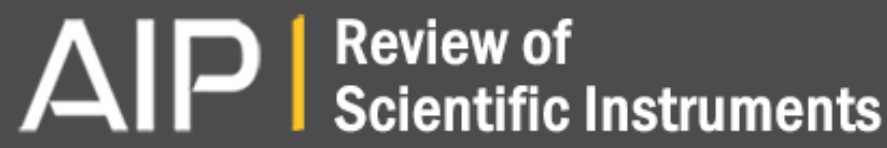

In-vessel calibration of the imaging diagnostics for the real-time protection of the JET ITER-like wall

V. Huber, A. Huber, D. Kinna, I. Balboa, S. Collins, N. Conway, P. Drewelow, C. F. Maggi, G. F. Matthews, A.

G. Meigs, Ph. Mertens, M. Price, G. Sergienko, S. Silburn, A. Wynn, and K.-D. Zastrow

Citation: Review of Scientific Instruments 87, 11D430 (2016); doi: 10.1063/1.4959912

View online: http://dx.doi.org/10.1063/1.4959912

View Table of Contents: http://scitation.aip.org/content/aip/journal/rsi/87/11 ?ver=pdfcov

Published by the AIP Publishing

\section{Articles you may be interested in}

Publisher's Note: "In-vessel calibration of the imaging diagnostics for the real-time protection of the JET ITERlike wall” [Rev. Sci. Instrum. 87, 11D430 (2016)]

Rev. Sci. Instrum. 87, 11F903 (2016); 10.1063/1.4962987

Invited Article: A novel calibration method for the JET real-time far infrared polarimeter and integration of polarimetry-based line-integrated density measurements for machine protection of a fusion plant

Rev. Sci. Instrum. 86, 091301 (2015); 10.1063/1.4929443

Upgrade of the infrared camera diagnostics for the JET ITER-like wall divertora)

Rev. Sci. Instrum. 83, 10D530 (2012); 10.1063/1.4740523

A protection system for the JET ITER-like wall based on imaging diagnosticsa)

Rev. Sci. Instrum. 83, 10D727 (2012); 10.1063/1.4738742

Implementation of an in-vessel calibration light source for JETa)

Rev. Sci. Instrum. 83, 10D505 (2012); 10.1063/1.4729502

\section{High Peak Power Femtosecond Lasers}

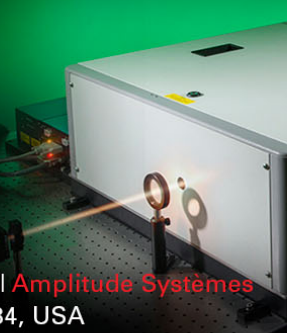




\title{
In-vessel calibration of the imaging diagnostics for the real-time protection of the JET ITER-like wall
}

\author{
V. Huber, ${ }^{1, a)}$ A. Huber, ${ }^{2}$ D. Kinna,${ }^{3}$ I. Balboa,${ }^{3}$ S. Collins,${ }^{3}$ N. Conway, ${ }^{3}$ P. Drewelow, ${ }^{4}$ \\ C. F. Maggi, ${ }^{3}$ G. F. Matthews, ${ }^{3}$ A. G. Meigs, ${ }^{3}$ Ph. Mertens, ${ }^{2}$ M. Price,${ }^{3}$ G. Sergienko, ${ }^{2}$ \\ S. Silburn, ${ }^{3}$ A. Wynn, ${ }^{5}$ and K.-D. Zastrow ${ }^{3}$ \\ ${ }^{1}$ Forschungszentrum Jülich GmbH, Supercomputing Centre, 52425 Jülich, Germany \\ ${ }^{2}$ Forschungszentrum Jülich GmbH, Institut für Energie- und Klimaforschung-Plasmaphysik, \\ Partner of the Trilateral Euregio Cluster (TEC), 52425 Jülich, Germany \\ ${ }^{3}$ CCFE, Culham Science Centre, Abingdon OX14 3DB, United Kingdom \\ ${ }^{4}$ MPI für Plasmaphysik, Greifswald, Germany \\ ${ }^{5}$ York Plasma Institute, University of York, Heslington, York YO10 5DD, United Kingdom
}

(Presented 6 June 2016; received 3 June 2016; accepted 28 June 2016; published online 23 August 2016; publisher error corrected 26 August 2016)

\begin{abstract}
The in situ absolute calibration of the JET real-time protection imaging system has been performed for the first time by means of radiometric light source placed inside the JET vessel and operated by remote handling. High accuracy of the calibration is confirmed by cross-validation of the near infrared (NIR) cameras against each other, with thermal IR cameras, and with the beryllium evaporator, which lead to successful protection of the JET first wall during the last campaign. The operation temperature ranges of NIR protection cameras for the materials used on JET are Be $650-1600^{\circ} \mathrm{C}, \mathrm{W}$ coating 600-1320 ${ }^{\circ} \mathrm{C}$, and W $650-1500{ }^{\circ} \mathrm{C}$. [http://dx.doi.org/10.1063/1.4959912]
\end{abstract}

\section{INTRODUCTION}

In JET with ITER-like wall (JET-ILW), the first wall was changed to metallic materials (tungsten and beryllium) which require a reliable protection system because too high temperatures of the plasma-facing components (PFCs) can result in beryllium melting or thermal fatigue of tungsten. The protection system based on imaging diagnostics has been implemented on JET in $2011^{1,2}$ and was at this time calibrated in the laboratory by using a calibrated blackbody. Then the calibration was corrected taking into the account the transmittance of the in- and ex-vessel optical components. It has become clear that this calibration method cannot provide the required accuracy of 5\% for first wall protection. Therefore, the in-vessel calibration of the imaging systems has been performed for the first time in 2015 with the help of a radiometric light source which has been placed inside the JET vacuum vessel by means of the remote-handling arm (RHA). In this contribution, we will present the calibration process of near infrared (NIR) cameras, the new calibration software, temperature measurements of the protection system, and their validation.

\section{JET PROTECTION IMAGING SYSTEM}

JET imaging systems contain four wide angle views, four tangential divertor views, and two top views of the divertor covering $25 \%-66 \%$ of the main chamber wall and up to $43 \%$ of

Note: Contributed paper, published as part of the Proceedings of the 21st Topical Conference on High-Temperature Plasma Diagnostics, Madison, Wisconsin, USA, June 2016.

a) Author to whom correspondence should be addressed. Electronic mail: V.Huber@fz-juelich.de. the divertor. ${ }^{1}$ Analog monochrome CCD cameras (HITACHI $\mathrm{KP} / \mathrm{M} 1 \mathrm{AP}$ ) operating in the near infrared wavelength region are used to measure the surface temperature of the PFCs. This type of camera demonstrates an acceptable reliability in the JET environment because of their operation robustness under high magnetic field and their resistance to neutrons and gamma radiation. The imaging sensors are equipped with near infrared (NIR) filters. Two types of band pass interference filters (IFs) have been used: $\lambda 980 \pm 10 \mathrm{~nm}$ and $\lambda 1016 \pm 40 \mathrm{~nm}$.

A frame grabber converts the analog output of the CCD camera into a digital, 8-bit, gigabit Ethernet signal which is distributed to three different systems by an Ethernet switch: (1) the real time processing unit (RTPU), (2) the data capture and replay, and (3) the live display. The RTPU is a field programmable gate array (FPGA) board that performs the Regions of Interest (ROI) analysis. Thus, Regions of Interest (ROI) are analyzed in real time and the maximum temperature measured in each ROI is sent to the vessel thermal map (VTM) which is used by the plasma control system.

\section{CALIBRATION OF PROTECTION CAMERAS}

The calibration of the imaging systems has been performed with the help of integrating spheres coupled to a stable, broad spectrum light source of known radiance. The in-vessel calibration light source (ICLS) with a 4 in. diameter exit port and four lamps, ${ }^{3}$ developed at Oak Ridge National Laboratory, has been positioned by RHA inside the JET vacuum vessel.

Fig. 1 shows the spectral radiance, $\mathrm{L}_{\lambda}$, of the sphere during the operation of one lamp. It has the maximum in the NIR wavelength range and is thus best suited for calibration of protection cameras. The arrows indicate the location of the central wavelength of the used interference filters. The 


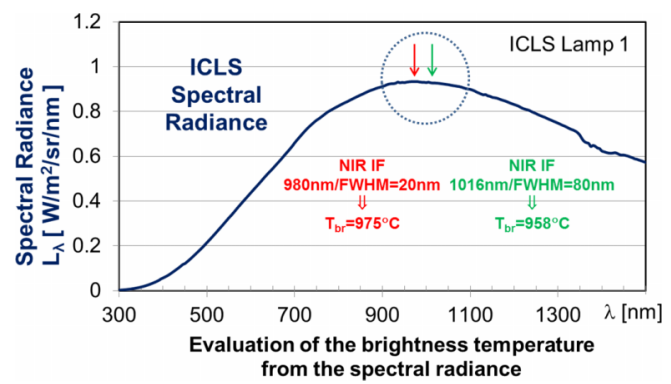

FIG. 1. Spectral radiance of the ICLS with Lamp 1.

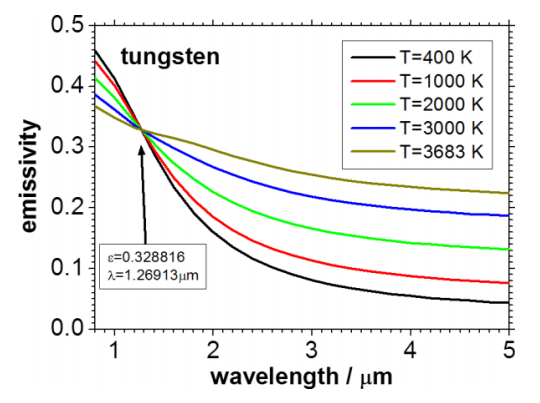

FIG. 3. W spectral emissivity according to Ref. 4.
$\mathrm{L}_{\lambda}$ at these wavelengths, $980 \mathrm{~nm}$ and $1016 \mathrm{~nm}$, correspond to the brightness temperatures, $\mathrm{T}_{\mathrm{br}}$, of $975^{\circ} \mathrm{C}$ and $958^{\circ} \mathrm{C}$. Additionally, the integrated sphere under different operation modes (different lamps or a combination of the lamps) has been cross-calibrated in the lab against a Pegasus R blackbody with the emissivity of $\varepsilon=0.995$ controlled by a thermocouple with an uncertainty of $\pm 2{ }^{\circ} \mathrm{C},{ }^{4}$ which was additionally crossvalidated with a one-color $\left(\lambda_{\text {eff }}=980 \mathrm{~nm}\right)$ pyrometer. Fig. 2 shows the digital levels measured by a camera as a function of the temperature of the blackbody. The experimental results fit well with the Planck radiation function (see Fig. 3). The points represent the mean values and the error bars are the standard deviations measured in a region of interest of a frame averaged over 100 frames. Red symbols are the results by using the ICLS. The temperature uncertainty by using the direct calculation of the $\mathrm{T}_{\mathrm{br}}$ from spectral radiance and by using the comparison with blackbody is below 5\%. Acquired brightness temperatures have been used for in situ calibration of all protection cameras. As a result of the calibration, the operation temperature ranges for the materials used on JET are $\mathrm{Be} 650-1600^{\circ} \mathrm{C}$, W coatings $600-1320^{\circ} \mathrm{C}$, and $\mathrm{W} 650-$ $1500^{\circ} \mathrm{C}$.

\section{SPECTRAL EMISSIVITY OF JET MATERIALS}

The JET main chamber is protected by guard limiters covered with castellated beryllium tiles. Furthermore, the divertor horizontal targets are constituted of bulk tungsten lamellas whereas the vertical targets are CFC coated tiles with a $12-20 \mu \mathrm{m}$ tungsten layer. For surface temperature measurements with high accuracy, as required for the JET wall protection, the knowledge of the spectral emissivity for each material used is essential.

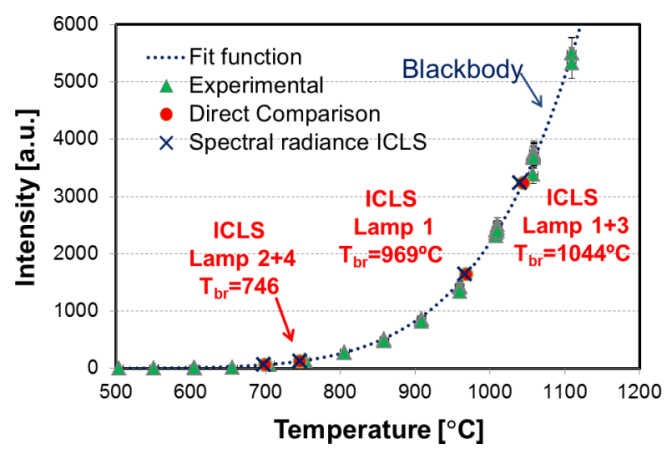

FIG. 2. Experimental results fitted by Planck radiation function.
The tungsten spectral emissivity, shown in Fig. 3 for different surface temperatures, is well documented. ${ }^{5}$ The so-called "X-point of W," where the emissivity wavelength isotherms cross, corresponds to $\lambda \approx 1.27 \mu \mathrm{m}$. The usage of the cameras equipped with IFs at the central wavelengths of $980 \mathrm{~nm}$ and $1016 \mathrm{~nm}$, close to the "X-point," brings the advantage of a weak dependence of $\varepsilon_{\lambda}$ on temperature. In contrary to the bulk tungsten, additional research to determine the emissivities of beryllium and of the tungsten coatings was required. The emissivity of the $\mathrm{W}$ coatings deposited on Dunlop DMS 780 was investigated in the range of $700-1200{ }^{\circ} \mathrm{C}^{6}$ using a steady state heating regime. A value of $0.63 \pm 0.07$ at $\lambda=1064 \mathrm{~nm}$ was found for $10 \mu \mathrm{m}$ thickness, while for $20 \mu \mathrm{m}$, the spectral emissivity was $0.59 \pm 0.06$. This spread of values is due to the structure of the CFC substrate. In the temperature range of $700-1200{ }^{\circ} \mathrm{C}$, there is no significant dependence of the $\mathrm{W}$ coatings emissivity on the surface temperature. Additionally, beryllium emissivity measurements on the JET neutral beam test bed have been performed. The values of emissivity used for the protection NIR imaging cameras (with IF $1016 \pm 40 \mathrm{~nm}$ ) are bulk tungsten $\varepsilon=0.42-1.98 \times 10^{-5} \times \mathrm{T}(\mathrm{K}), \mathrm{W}$ coatings $\varepsilon=0.6$, and beryllium $\varepsilon=0.25$.

\section{CALIBRATION SOFTWARE}

Because the calibration activities with RHA are restricted in time, very expensive, and cannot be repeated, special software for the data acquisition and quick data analysis is required. A new powerful software package calib has been developed and successfully used during the calibration of the protection as well as of visual and Mid-wavelength infrared (MWIR) imaging systems. The calibration software consists of several components written in Python on the basis of the new developed framework JUVIL (JET Users Video Imaging Library),${ }^{7}$ which provides an easy access to the video data of different cameras installed at JET.

The calibration editor caledit enables rapid data acquisition during in situ calibration and experiments in the lab. It stores the calibration settings for each measurement, triggers offline pulses for experiments in the lab, displays corresponding local or remote videos, and allows quick analysis of measured data. The calibration viewer calview displays the list of performed calibration measurements for a specific camera and experiment, loads corresponding videos, and provides the editors in order to modify the calibration 


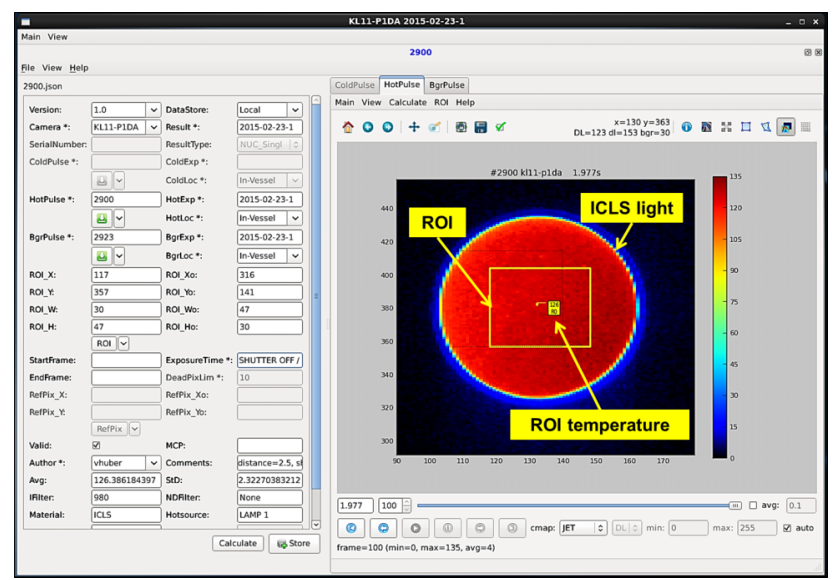

FIG. 4. Calibration software for data evaluation.

settings. The data evaluation is done by calib and calres components. Calib allows the selection of a ROI and of a corresponding background pulse for each measurement and stores them together with the calculated ROI's average and standard deviation, and, for MWIR cameras, also with the gain, offset, and dead pixels, as intermediate results (see Fig. 4). Calres analyses the selected intermediate data and produces the final results: for the protection cameras, the experimental data fitted by Planck radiation function and calibration curves for beryllium, bulk W, and W coatings; and for visual cameras, the filter calibration factors.

All calibration settings and results are stored in the appropriate format in the local or remote database. The advantage of such an approach is the traceability of the calibration procedure from the beginning to the end and the possibility of quick re-calculation of the calibration results if any parameters are changed.

\section{RESULTS AND DISCUSSION}

One example of the calibration of the wide angle KL14 camera by ICLS is shown in Fig. 5: digital levels measured by the camera versus brightness temperatures of the ICLS with one lamp and with combination of two lamps. The Planck radiation curve was used as a fit of the experimental data. The small spread of experimental values is due to the accuracy of the transmittance values of the neutral filters (about 2\%) used during the calibration. The offset value of the fit function,

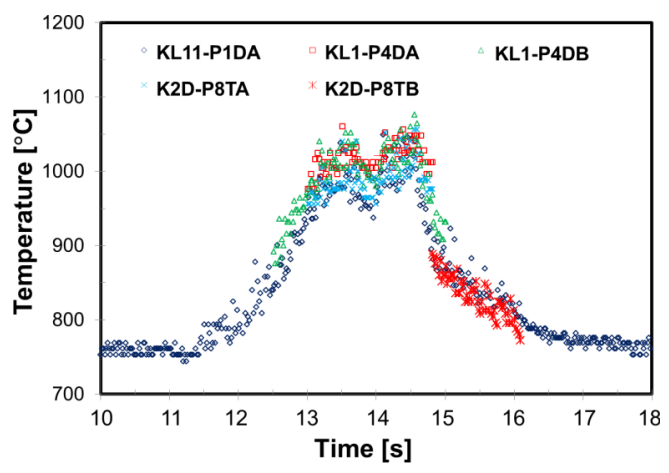

FIG. 6. Time traces of $\mathrm{T}_{\text {surf }}$ measured by different NIR cameras.

one of the fit parameters, is nearly zero, pointing to the good quality of the fit. The calibration curve could be easily adapted to the wanted material as shown in Fig. 5: yellow for tungsten coatings, red for bulk tungsten, and green for beryllium. On the right hand side is shown the Field of View (FoV) of this camera.

In order to confirm the calibration of the protection cameras, the response of the protection system to main chamber and divertor hot spots has been commissioned during the JET plasma restart. Validation of the temperature outputs from the RTPU has been performed by cross comparison of the protection cameras against each other. Fig. 6 shows the comparison of the surface temperature of the bulk tungsten measured by protection cameras during the plasma discharge demonstrating a relatively good agreement.

Up to $800^{\circ} \mathrm{C}$, the protection camera overestimates the temperature due to continuum radiation. Note that all these cameras located in different toroidal locations and the deviations could be explained by discrepancies in the toroidal symmetry. Because of the small dynamic ranges of the protection cameras, we are using several cameras which are adjusted for the different temperature windows. Additionally, the temperatures measured by protection cameras were compared with the thermal IR camera (at the $4-\mu \mathrm{m}$ wavelength) ${ }^{8}$ as shown in Fig. 7. The comparison was done under consideration of spatial and time resolution of the compared cameras. Above $800^{\circ} \mathrm{C}$, the agreement between the protection cameras and the thermal IR camera is within $5 \%$ on average.

The validation of the wide angle cameras in the main chamber has been done during the discharge phase with an inner wall MARFE. We used the fact that the MARFE

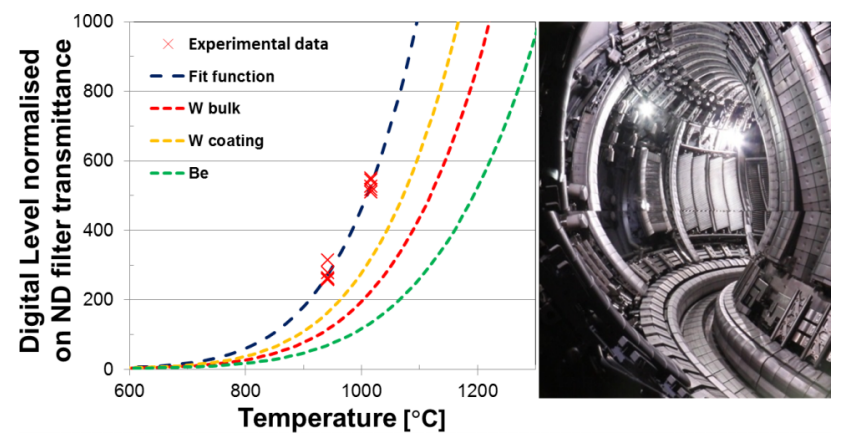

FIG. 5. Wide angle camera calibration results.

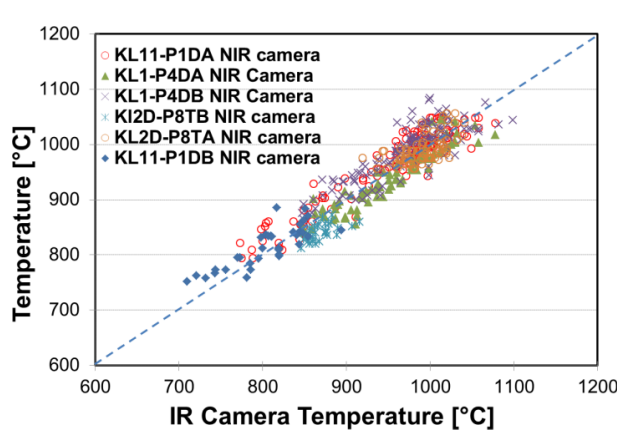

FIG. 7. $\mathrm{T}_{\text {surf }}{ }^{\mathrm{NIR}}$ versus $\mathrm{T}_{\text {surf }}{ }^{\mathrm{IR}}$. 


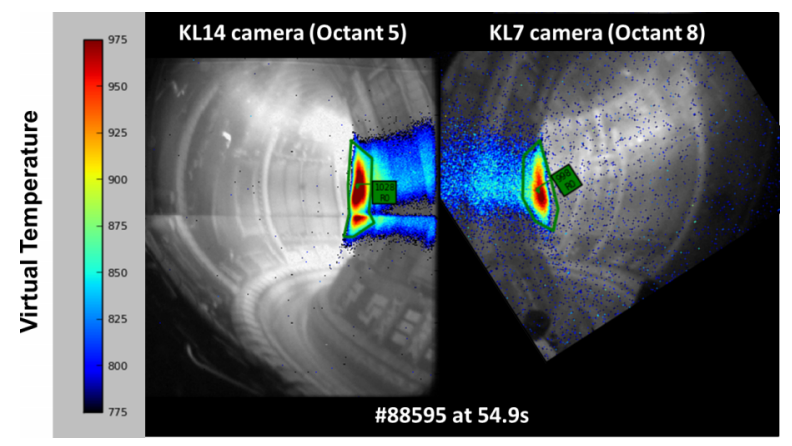

FIG. 8. Temperature validation during inner wall MARFE phase.

provides an excellent toroidal symmetry with strong continuum radiation in NIR range. The relative standard deviation of the "virtual" temperatures delivered by JET wide angle cameras is below $4 \%$, confirming the reliability of the invessel calibration (see Fig. 8). Also the cross-validation NIR cameras with the beryllium evaporator, equipped with a thermocouple, demonstrates the good agreement within $20^{\circ} \mathrm{C}$ at $\mathrm{T}_{\text {surf }}=900^{\circ} \mathrm{C}$.

\section{CONCLUSION}

The calibration of the imaging systems has been performed with the help of an in-vessel radiometric light source which has been controlled inside the JET vacuum vessel by means of the remote-handling arm. The absolutely calibrated protection system based on the imaging diagnostics was timely validated and commissioned during the restart phase and beginning of the 2015 scientific campaign. It has already successfully led to a safe landing of the plasma when hot spots were observed on the first JET wall and the discharge had to be switched off.

\section{ACKNOWLEDGMENTS}

This work has been carried out within the framework of the Contract for the Operation of the JET Facilities and has received funding from the European Union's Horizon 2020 research and innovation programme. The views and opinions expressed herein do not necessarily reflect those of the European Commission.

${ }^{1}$ G. Arnoux et al., Rev. Sci. Instrum. 83, 10D727 (2012).

${ }^{2}$ A. Huber et al., Rev. Sci. Instrum. 83, 10D511 (2012).

${ }^{3}$ T. M. Biewer et al., Rev. Sci. Instrum. 83, $10 \mathrm{D} 505$ (2012).

${ }^{4}$ See http://www.isotech.co.uk/infrared-calibrators/high-emissivity-blackbo dy-sources/pegasus-r-model-97 for information about Pegasus R blackbody source.

${ }^{5}$ CRC Handbook of Chemistry and Physics, 75 th ed., edited by D. R. Lide and H. P. R. Frederikse (CRC, 1994).

${ }^{6} \mathrm{C}$. Ruset et al., "Advanced calibration of the PIW IR camera," JET Progress Report, JW10-TA-PIW-ACIR-02 (2012).

${ }^{7}$ V. Huber et al., "JUVIL-New software for JET cameras," JET Report No. NJOC-PR(16) 15114

${ }^{8}$ I. Balboa et al., Rev. Sci. Instrum. 87, 11D419 (2016). 